\title{
FAITHFUL ABELIAN GROUPS OF INFINITE RANK
}

\author{
ULRICH ALBRECHT
}

(Communicated by Bhama Srinivasan)

\begin{abstract}
Let $B$ be a subgroup of an abelian group $G$ such that $G / B$ is isomorphic to a direct sum of copies of an abelian group $A$. For $B$ to be a direct summand of $G$, it is necessary that $G$ be generated by $B$ and all homomorphic images of $A$ in $G$. However, if the functor $\operatorname{Hom}(A,-)$ preserves direct sums of copies of $A$, then this condition is sufficient too if and only if $M \otimes_{E(A)} A$ is nonzero for all nonzero right $E(A)$-modules $M$. Several examples and related results are given.
\end{abstract}

1. Introduction. There are only very few criteria for the splitting of exact sequences of torsion-free abelian groups. The most widely used of these was given by Baer in 1937 [F, Proposition 86.5]:

If $C$ is a pure subgroup of a torsion-free abelian group $G$, such that $G / C$ is homogeneous completely decomposable of type $\tau$, and all elements of $G \backslash C$ are of type $\tau$, then $C$ is a direct summand of $G$.

Because of its numerous applications, many attempts have been made to extend the last result to situations in which $G / C$ is not completely decomposable. Arnold and Lady succeeded in 1975 in the case that $G$ is torsion-free of finite rank.

Before we can state their result, we introduce some additional notation: Suppose that $A$ and $G$ are abelian groups. The $A$-socle of $G$, denoted by $S_{A}(G)$, is the subgroup of $G$ generated by $\{\phi(A) \mid \phi \in \operatorname{Hom}(A, G)\}$. The abelian group $G$ is $A$-projective if it is isomorphic to a direct summand of $\bigoplus_{I} A$ for some index-set $I$. If $I$ can be chosen to be finite, then $G$ has finite $A$-rank. Finally, $A$ is faithful if $I A \neq A$ for all proper right ideals $I$ of the endomorphism ring, $E(A)$, of $A$.

Arnold and Lady showed in [AL, Theorem 2.1]: A torsion-free abelian group $A$ of finite rank is faithful iff every exact sequence $0 \rightarrow B \stackrel{\alpha}{\rightarrow} G \stackrel{\beta}{\rightarrow} P \rightarrow 0$ of torsionfree abelian groups of finite rank with $G=\left\langle\alpha(B), S_{A}(G)\right\rangle$ and $P A$-projective is split-exact, where $\left\langle\alpha(B), S_{A}(G)\right\rangle$ denotes the subgroup of $G$ which is generated by $\alpha(B)$ and $S_{A}(G)$.

Unfortunately, Arnold and Lady's proof uses induction on the rank of $P$ to show that faithfulness is sufficient for the splitting of the sequences under consideration. This method breaks down if $P$ has infinite $A$-rank. Nevertheless, Baer's classical theorem follows from Arnold and Lady's result in the finite rank case.

The goal of this paper is to discuss the class of abelian groups $A$ such that every exact sequence $0 \rightarrow B \stackrel{\alpha}{\rightarrow} G \stackrel{\beta}{\rightarrow} P \rightarrow 0$ with $P A$-projective and $G=\left\langle S_{A}(G), \alpha(B)\right\rangle$

Received by the editors April 29, 1986 and, in revised form, December 10, 1986 and February 25, 1987.

1980 Mathematics Subject Classification (1985 Revision). Primary 20K20, 20K30, 16A65; Secondary 20K25, 16A50.

Key words and phrases. Endomorphism ring, faithful, Baer's lemma. 
splits. In order to remove the restrictions on the rank of $A$, we consider selfsmall abelian groups, i.e. groups $A$ such that, for every index-set $I$ and every $\phi \in \operatorname{Hom}\left(A, \bigoplus_{I} A\right)$, there is a finite subset $I^{\prime}$ of $I$ with $\phi(A) \subseteq \bigoplus_{I^{\prime}} A$. Obviously, a torsion-free group of finite rank is self-small, but the class of self-small groups contains, for instance, all abelian groups $A$ with $E(A)$ countable [AM, Corollary 1.4]. Finally, $A$ is fully faithful if $M \otimes_{E(A)} A \neq 0$ for all nonzero right $E(A)$-modules. $M$. The main result of this paper is

THEOREM 2.1. The following are equivalent for a self-small abelian group $A$ :

(a) $A$ is fully faithful.

(b) Every exact sequence $0 \rightarrow B \stackrel{\alpha}{\rightarrow} G \stackrel{\beta}{\rightarrow} P \rightarrow 0$ with $P$ A-projective and $G=\left\langle\alpha(B), S_{A}(G)\right\rangle$ splits.

$\S 3$ will provide various examples that illustrate the previous results. In particular, $A$ is self-small and faithful if $E(A)$ is a commutative or right principal subring of a finite dimensional Q-algebra, or $A$ is an $E$-ring.

2. Fully faithful abelian groups. Since we frequently use the basic properties of self-small abelian groups which have been discussed in [AM], we shall begin with a short summary of these. For reasons of simplicity, we denote the functor $\operatorname{Hom}(A,-)$ by $H_{A}$ and the functor $-\otimes_{E(A)} A$ by $T_{A}$, where $A$ is an abelian group. If $G$ is an abelian group, and $M$ is a right $E(A)$-module, then there is a map $\theta_{G}: T_{A} H_{A}(G) \rightarrow G$ defined by $\theta_{G}(\phi \otimes a)=\phi(a)$ and a map $\phi_{M}: M \rightarrow H_{A} T_{A}(M)$ defined by $\left[\phi_{M}(m)\right](a)=m \otimes a$ for all $\phi \in H_{A}(G), m \in M$, and $a \in A$. In the case that $A$ is self-small, $\theta_{G}$ is an isomorphism for all $A$-projective groups, while $\phi_{M}$ is an isomorphism for all projective right $E(A)$-modules $M$.

THEOREM 2.1. The following are equivalent for a self-small abelian group $A$ :

(a) $A$ is fully faithful.

(b) Every exact sequence $0 \rightarrow B \stackrel{\alpha}{\rightarrow} G \stackrel{\beta}{\rightarrow} P \rightarrow 0$ of abelian groups with $P$ $A$-projective and $G=\left\langle\alpha(B), S_{A}(G)\right\rangle$ splits.

ProOF. (a) $\Rightarrow(\mathrm{b})$. Consider an exact sequence

$$
0 \rightarrow B \stackrel{\alpha}{\rightarrow} G \stackrel{\beta}{\rightarrow} P \rightarrow 0
$$

of abelian groups with $P A$-projective and $G=\left\langle\alpha(B), S_{A}(G)\right\rangle$. It induces the exact sequence

$$
0 \rightarrow H_{A}(B) \rightarrow H_{A}(G) \rightarrow M \rightarrow 0
$$

of right $E(A)$-modules where $M=\operatorname{im} H_{A}(\beta)$ is a submodule of the projective right $E(A)$-module $H_{A}(P)$.

To insure the splitting of (E2), it suffices to show $M=H_{A}(P)$. For this, define a map $\theta: T_{A}(M) \rightarrow P$ by $\theta(\phi \otimes a)=\phi(a)$ for all $\phi \in M$ and $a \in A$. An application of $T_{A}$ to (E2) yields the top row of the commutative diagram

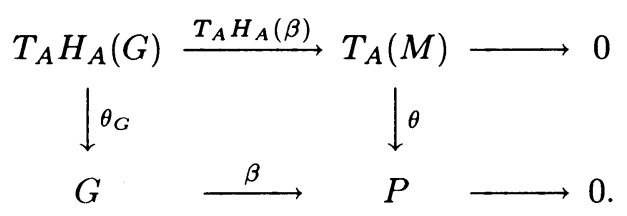


Since $S_{A}(G)=\operatorname{im} \theta_{G}$ and $G=\left\langle\alpha(B), S_{A}(G)\right\rangle$, the map $\beta \theta_{G}=\theta T_{A} H_{A}(\beta)$ is onto. Therefore, $\theta$ is onto. Furthermore, the inclusion map $i: M \rightarrow H_{A}(P)$ induces the commutative diagram

$$
\begin{aligned}
& T_{A}(M) \stackrel{T_{A}(i)}{\longrightarrow} T_{A} H_{A}(P) \longrightarrow T_{A}\left(H_{A}(P) / M\right) \longrightarrow 0 \\
& T_{A}(M) \stackrel{\theta}{\longrightarrow} \quad P \quad \longrightarrow 0 .
\end{aligned}
$$

The map $\theta_{P}$ is an isomorphism since $A$ is self-small. Consequently, $T_{A}(i)$ is onto. Therefore, $T_{A}\left(H_{A}(P) / M\right)=0$. By (a), $M=H_{A}(P)$.

Let $\phi: H_{A}(P) \rightarrow H_{A}(G)$ be a splitting map for $H_{A}(\beta)$. An application of the functor $T_{A}$ yields the commutative diagram

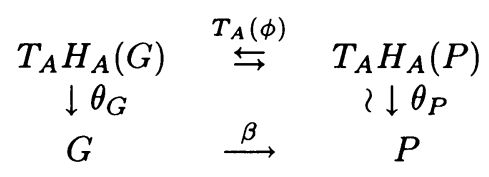

If $\nu=\theta_{G} T_{A}(\phi) \theta_{P}^{-1}$ then $\beta \nu=\mathrm{id}_{P}$, and (E1) splits.

(b) $\Rightarrow$ (a). Let $M$ be a right $E(A)$-module with $T_{A}(M)=0$. Choose a free resolution $0 \rightarrow U \stackrel{\alpha}{\rightarrow} F \stackrel{\beta}{\rightarrow} M \rightarrow 0$ where $F$ is a free right $E(A)$-module. It induces the exact sequence $T_{A}(U) \stackrel{T_{A}(\alpha)}{\rightarrow} T_{A}(F) \rightarrow 0$ (E2). The $E(A)$-module $U$ admits an exact sequence $P \stackrel{\delta}{\rightarrow} U \rightarrow 0$ with $P$ projective. The exactness of the sequence $T_{A}(P) \stackrel{T_{A}(\delta)}{\rightarrow} T_{A}(U) \rightarrow 0$ yields an epimorphism $T_{A}(\alpha \delta): T_{A}(P) \rightarrow T_{A}(F)$ which splits by (b). Consequently, the top row of the commutative diagram

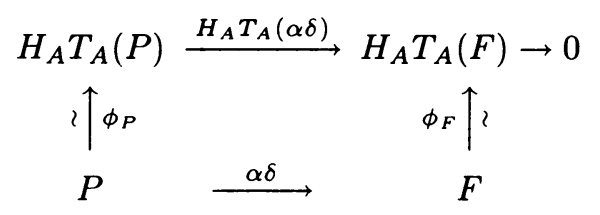

is exact. Hence, $\alpha \delta$ is onto, and the same holds for $\alpha$. But this is only possible if $M=0$.

A slight modification of the proof of the last result yields

COROLLARY 2.2. The following are equivalent for a self-small abelian group $A$ :

(a) $A$ is faithful.

(b) $T_{A}(M) \neq 0$ for all finitely generated nonzero right $E(A)$-modules $M$.

(c) Every exact sequence $0 \rightarrow B \stackrel{\alpha}{\rightarrow} G \stackrel{\beta}{\rightarrow} P \rightarrow 0$ of abelian groups with $G=$ $\left\langle\alpha(B), S_{A}(G) \rightarrow\right.$ and $P$ A-projective of finite $A$-rank splits.

PROOF. (c) $\Rightarrow($ b) is shown exactly as in the proof of Theorem 2.1 once we have observed that $F$ can be chosen to be finitely generated.

(b) $\Rightarrow$ (a) is obvious since being faithful is equivalent to satisfying $T_{A}(M) \neq 0$ for all cyclic right $E(A)$-modules $M$.

(a) $\Rightarrow$ (c) is shown as in the proof of $[\mathbf{A L}$, Theorem 2.1] by induction on the $A$-rank of $P$. 
This last result contains [AL, Theorem 2.1] since every torsion-free group of finite rank is self-small. Moreover, if $A$ is torsion-free of finite rank, then an $A$-projective group $P$ has finite $A$-rank exactly if it has finite torsion-free rank.

Furthermore, if $A$ is flat as an $E(A)$-module, then a right $E(A)$-module $M$ satisfies $T_{A}(M)=0$ iff $T_{A}(U)=0$ for all finitely generated submodules $U$ of $M$. Consequently, faithful and fully faithful are the same in this case:

COROLLARY 2.3. The following are equivalent for a self-small abelian group $A$ which is flat as an $E(A)$-module.

(a) $A$ is faithful.

(b) Every exact sequence $0 \rightarrow B \stackrel{\alpha}{\rightarrow} G \stackrel{\beta}{\rightarrow} P \rightarrow 0$ with $P$ A-projective and $G=\left\langle\alpha(B), S_{A}(G)\right\rangle$ splits.

3. Examples. In $[\mathbf{A 2}]$, we considered the property in Theorem 2.1(b) in conjunction with the following property: Every subgroup $B=S_{A}(B)$ of an $A$-projective group is $A$-projective. We showed that a self-small abelian group $A$ has these two properties exactly if it is flat as an $E(A)$-module and has a right hereditary endomorphism ring. Consequently, we obtain

PROPOSITION 3.1. A self-small abelian group $A$ which is flat as an $E(A)$ module and has a right hereditary endomorphism ring is fully faithful.

The conditions of Proportion 3.1 are satisfied for instance if $A$ is a torsion-free reduced abelian group with a two-sided Noetherian hereditary endomorphism ring [A1, Proposition 3.2].

However, there are examples of faithful abelian groups which do not have hereditary endomorphism rings:

A ring $R$ is an $E$-ring if every $\mathbf{Z}$-endomorphisms of $R^{+}$is multiplication by some element of $R$. $E$-rings were introduced by Schultz in $[\mathbf{S}]$ and Beaumont and Pierce in $[\mathbf{B P}]$.

Let $\Pi$ denote the set of primes of $\mathbf{Z}$. The ring $E=\Pi_{p \in \Pi} \mathbf{Z} / p \mathbf{Z}$ is an $E$-ring. It is self-injective since $\mathbf{Z} / p \mathbf{Z}$ is self-injective, and the class of self-injective rings is closed under products. Osofsky showed in $[\mathbf{O}]$ that a self-injective hereditary ring is semisimple Artinian. Therefore, $E$ is an example of a nonhereditary $E$-ring.

EXAMPLE 3.2. Every $E$-ring $R$ is self-small, fully faithful, and flat as an $R$ module.

PROOF. Clearly, $R^{+}$is isomorphic to $R$ as an $R$-module. Thus, $M \otimes_{R} R \cong M$, and $R^{+}$is faithful and flat as an $R$-module. Furthermore, if $\phi \in \operatorname{Hom}\left(R, \bigoplus_{I} R\right)$, then there are $r_{i} \in R$ for $i \in I$ with $\phi(r)=\left(r_{i} r\right)_{i \in I}$. Moreover, $\phi(1)=\left(r_{i}\right)_{i \in I} \in$ $\bigoplus_{I} R$ and hence $r_{i}=0$ for almost all $i \in I$. Thus $R$ is self-small.

Another class of examples is given by the next two results.

Proposition 3.3. A torsion-free abelian group $A$ such that $E(A)$ is a right principal ideal ring, whose additive group has finite rank, is self-small and faithful.

Proof. Since $E(A)$ is countable, it remains to show that $A$ is faithful. Let $I=\phi E(A)$ be a right ideal with $I A=A$. Consider the exact sequence $0 \rightarrow U \stackrel{\nu}{\rightarrow}$ $A \stackrel{\phi}{\rightarrow} A \rightarrow 0$. An application of the functor $\operatorname{Hom}(-, A)$ yields the exact sequence

$$
0 \rightarrow E(A) \stackrel{\mathrm{Hom}(\phi, A)}{\rightarrow} E(A) \stackrel{\mathrm{Hom}(\nu, A)}{\rightarrow} M \rightarrow 0
$$


of left $E(A)$-modules where $M=\operatorname{im}(\operatorname{Hom}(\nu, A))$ is a submodule of $\operatorname{Hom}(U, A)$ which is torsion-free as an abelian group. Because of $r_{0}(E(A))<\infty$, we have $M=0$. Consequently, $\operatorname{Hom}(\phi, A)$ is an isomorphism. An application of the functor $\operatorname{Hom}_{E(A)}(-, A)$ yields the commutative diagram

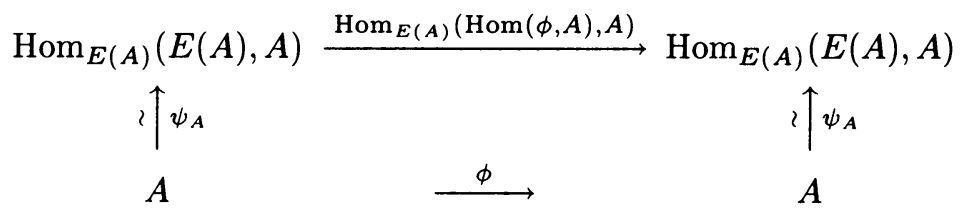

where the map $\psi_{A}$, which is defined by $\left[\psi_{A}(a)\right](\alpha)=\alpha(a)$ for all $\alpha \in E(A)$ and $a \in A$, is an isomorphism. Thus, $\phi$ is an isomorphism, and $I=E(A)$.

THEOREM 3.4. A torsion-free reduced abelian group $A$ such that $\mathbf{Q} \otimes_{\mathbf{z}} E(A)$ is a commutative finite dimension $\mathbf{Q}$-algebra is self-small and faithful.

ProOF. As in Proposition 3.3, it suffices to show that $A$ is faithful. Let $I$ be a right ideal of $E(A)$ with $A=I A$. If $E(A) / I$ is torsion, then there is a nonzero integer $m$ with $m E(A) \subseteq I$. Consider the descending chain

$$
I / m E(A) \supseteq\left(I / m^{2} E(A)\right) \supseteq \cdots \supseteq\left(I / m^{n} E(A)\right) \supseteq \cdots
$$

of right ideals of the finite ring $E(A) / m E(A)$. There is some $n<\omega$ with

$$
(I / m E(A))^{n}=(I / m E(A))^{n+1} .
$$

By [Ar1, Lemma 5.8], there exists some $y \in I$ such that

$$
(1+y+m E(A))(I / m E(A))^{n}=0 .
$$

In particular,

$$
(1+y+m E(A))\left(\left\langle I^{n}, m E(A)\right\rangle / m E(A)\right)=0 .
$$

This yields $(1+y) I^{n} \subseteq m E(A)$. Consequently, $m A \supseteq(1+y) I^{n} A=(1+y)(A)$ because of $I A=A$. For $a \in A$, choose $a^{\prime} \in A$ with $(1+y)(a)=m a^{\prime}$ and define $\phi(a)=m a^{\prime}$. Then, $1+y=m \phi \in m E(A) \subseteq I$. Hence, $1 \in I$ and $I=E(A)$.

If $E(A) / I$ is not torsion, then $\mathbf{Q} \otimes_{\mathbf{z}} I$ is a proper ideal of $\mathbf{Q} \otimes_{\mathbf{z}} E(A)$. If we can show that every proper ideal of $\mathbf{Q} \otimes_{\mathbf{z}} E(A)$ has a nonzero left annihilator, then there exists a nonzero integer $m$ and $0 \neq \phi \in E(A)$ with $(1 / m \otimes \phi)(\mathbf{Q} \otimes \mathbf{z} I)=0$. Thus, $0=\phi i A=\phi(A)$, a contradiction.

The ring $\mathbf{Q} \otimes_{\mathbf{z}} E(A)$ is Artinian. Suppose that there exists a proper ideal $J$ of $\mathbf{Q} \otimes_{\mathbf{z}} E(A)$ which is minimal with respect to the property $\left\{\phi \in \mathbf{Q} \otimes_{\mathbf{z}} E(A) \mid \phi J=\right.$ $0\}=0$. If $J^{2} \subsetneq J$, then there is a nonzero element $\phi \in \mathbf{Q} \otimes_{\mathbf{z}} E(A)$ with $\phi J^{2}=0$. Because of $\phi J \neq 0$, we obtain that $J$ has a nonzero annihilator. The resulting contradiction shows $J^{2}=J$. By [Ar1, Lemma 5.8], there is $y \in J$ with $(1+y) J=0$ since $J$ is finitely generated. Since $J$ is proper, $1+y \neq 0$. Therefore, $J$ has a nonzero annihilator. The resulting contradiction proves the lemma.

COROLlARY 3.5. $(V=L)$. Let $\kappa$ be an uncountable cardinal number. There exists $2^{\kappa}$ many nonisomorphic self-small, fully faithful abelian groups of cardinality $\kappa$.

PROOF. Let $R$ be a torsion-free reduced ring as in Proposition 3.3 or Theorem 3.4. By [DG, Theorem 3.3], there are $2^{\kappa}$ many nonisomorphic abelian groups of 
cardinality $\kappa$ with $E(A) \cong R$ such that every finite subset of $A$ is contained in a free $E(A)$-submodule of $A$. The $R$-module $A$ is flat because it is the direct limit of its finitely generated free submodules. Combining Proposition 3.3 and Theorem 3.4 with Corollary 2.3 and Theorem 2.1 yields that each such $A$ is fully faithful.

Arnold has investigated torsion-free groups which are flat as an $E(A)$-modules [Ar , AL]. Lately, Goeters and Reid discovered an interesting characterization of abelian groups which are flat over their endomorphism ring and have finite rank [GR].

\section{BIBLIOGRAPHY}

[A1] U. Albrecht, A note on locally A-projective groups, Pacific J. Math. 120 (1985), 1-17.

[A2] _ Baer's Lemma and Fuchs' Problem 84a, Trans. Amer. Math. Soc. 293 (1986), 565-582.

[Ar1] D. M. Arnold, Finite rank torsion-free abelian groups and rings, Lecture Notes in Math., vol. 931, Springer-Verlag, Berlin, Heidelberg and New York, 1982.

[Ar2] _ Notes on abelian groups flat over $E(A)$, unpublished manuscript.

[AL] D. M. Arnold and E. L. Lady, Endomorphism rings and direct sums of torsion-free abelian groups, Trans. Amer. Math. Soc. 211 (1975), 225-236.

[AM] D. M. Arnold and C. E. Murley, Abelian groups, $A$, such that $\operatorname{Hom}(A,-)$ preserves direct sums of copies of $A$, Pacific J. Math. 56 (1975), 7-20.

[B] R. Baer, Abelian groups without elements of finite order, Duke Math. J. 3 (1937), 68-122.

[BP] R. A. Beaumont and R. S. Pierce, Torsion-free rings, Illinois J. Math. 5 (1961), 61-98.

[DG] M. Dugas and R. Göbel, Every cotorsion-free ring is an endomorphism ring, Proc. London Math. Soc. 45 (1982), 319-336.

[F] L. Fuchs, Infinite abelian groups, Vol. II, Academic Press, London and New York, 1973.

[GR] H. P. Goeters and J. R. Reid, On the $p$-ranks of $\operatorname{Hom}(A, B)$, preliminary report.

[O] B. L. Osofsky, Rings all of whose finitely generated modules are injective, Pacific J. Math. 14 (1964), 645-650.

[R] J. J. Rotman, Homological algebra, Academic Press, London and New York, 1982.

[S] P. Schultz, The endomorphism ring of the additive group of a ring, J. Austral. Math. Soc. 15 (1973), 60-69.

Department of Algebra, Combinatorics and Analysis, Division of Mathematics, Auburn University, Auburn, Alabama 36849 